\title{
The Challenges of Biopharmaceutical Products for Analytical Requirements
}

\section{Bruno Sarmento*}

Health Sciences Research Center, Department of Pharmaceutical Sciences, Instituto Superior de Ciências da Saúde - Norte, Rua Central de Gandra 1317, 4585-116 Gandra, Portugal

Biopharmaceuticals drugs are a class of therapeutics enclosing recombinant therapeutic proteins, engineered antibodies and genetic material. Biopharmaceuticals have acquired a key role in the treatment of various diseases such as diabetes, several types of cancer, and inflammatory diseases. They are attractive medicines in the medical point of view, but technologically they face major challenges, starting by their inapt chemical and physical instability. Forms of chemical degradation include deamidation, isomerization, hydrolysis, racemization, oxidation, disulfide formation and $\beta$-elimination. Physical stability is generally defined as the ability of a protein to retain at least its tertiary structure that is crucial for biological activity. Physical degradation involves reversible or irreversible denaturation through a loss of tertiary structure and unfolding with further reactions like chemical degradation, aggregation and precipitation. Side effects, immunogenicity, and allergic reactions have been caused when administering relatively impure or barely degraded preparations. To prevent such incidents, a strong awareness to attempt for highly pure and stable biopharmaceutical products has emerged. High purity begins with production and downstream processing of therapeutic proteins, mainly, and other biopharmaceutical agents. Only if the bulk material has an acceptable level of impurities, stable and robust formulations can be reasonably designed to preserve this state until the final administration to the patient [1].

Two main reasons, I believe, are on the basis of the challenges for establish accurate analytical requirements for fully characterize and control biopharmaceutical products: on the one hand, the microheterogeneity, comprising smallvariation in thechemical structure of biopharmaceutical molecule that does not result in a significant change in properties and bioactivity, limit the specificity of analytical methods; on the other hand, the absence of official monographs for new biopharmaceutical molecules force the biopharmaceutical companies to develop and validate new methodologies based on international guidelines, sometimes ambiguous for those less familiarized with regulatory biopharmaceutical science.

Efforts have been done by the International Conference of Harmonization ( $\mathrm{ICH})$ in the last few years in order to clarify the analytical analysis obligatory to biotechnological-based products. In special, guidelines focused on the purity of the drug substances. The Q3A (R2) guideline addresses the chemistry and safety aspects of impurities, including the listing of impurities in specifications and defines the thresholds for reporting, identification and qualification [2], The Q3B (R2) complements the guideline on impurities in new drug substances and provides advice in regard to impurities in products containing new drug substances. The guideline specifically deals with those impurities which might arise as degradation products of the drug substance or arising from interactions between drug substance and excipients or components of primary packaging materials, setting out a rationale for the reporting, identification and qualification of such impurities based on a scientific appraisal of likely and actual impurities observed, and of the safety implications, following the principles elaborated in the parent guideline [3].

Another relevant document is the Q5C, with the particular aspects of stability test procedures needed to take account of the special characteristics of products in which the active components are typically proteins and/or polypeptides [4].

But not only are the biopharmaceuticals as drug substances required to be verified before formulation, but also the final drug product due to the special storage needs that these products often demand. The robustness of the formulations against external stress factors is also important, because biopharmaceuticals are exposed to various types of stress during production, fill and finish, shipment, storage, and final administration. Among these stress factors are temperature changes, elevated temperature, freezing, thawing, mechanical stress, light exposure, or interaction with specific components derived from production equipment, primary packaging material, or administration devices used, all of which can potentially impede stability [1]. Denatured or aggregated protein species will not only be therapeutically inactive, but also may cause unpredictable side effects, such as immunogenicity or toxicity. In the same line, denaturation of genetic material or any other mean of destabilization leads to the loss of its biological activity. Moreover, siRNA molecules are neither trivial nor inexpensive, emphasizing the importance of their stability for the therapeutics. Thus, the analytical procedures developed to biopharmaceutical substances must reach the core of the formulation, and be robust enough to guarantee that the entire formulation is able to be characterized. Because of their importance to biopharmaceuticals stability analysis, analytical methodologies are used to examine protein and antibodies structure and interactions between biopharmaceuticals and excipients. Fourier-Transform Infrared (FTIR), circular dichroism, micro differential scanning calorimetry ( $\mu \mathrm{DSC}$ ) and western blot are examples of techniques explored to evaluate the molecular conformation.

Each biopharmaceutical must ensemble a tailor-made manufacturing process and formulation must be designed to meet

*Corresponding author: Bruno Sarmento, Health Sciences Research Center Department of Pharmaceutical Sciences, Instituto Superior de Ciências da Saúde - Norte, Rua Central de Gandra 1317, 4585-116 Gandra, Portugal, E-mail: bruno. sarmento@ff.up.pt

Received April 11, 2012; Accepted April 12, 2012; Published April 14, 2012

Citation: Sarmento B (2012) The Challenges of Biopharmaceutical Products for Analytical Requirements. Pharmaceut Anal Acta 3:e110. doi:10.4172/21532435.1000e110

Copyright: @ 2012 Sarmento B. This is an open-access article distributed under the terms of the Creative Commons Attribution License, which permits unrestricted use, distribution, and reproduction in any medium, provided the original author and source are credited. 
Citation: Sarmento B (2012) The Challenges of Biopharmaceutical Products for Analytical Requirements. Pharmaceut Anal Acta 3:e110. doi:10.4172/2153-2435.1000e110

Page 2 of 2

general requirements, such as the pharmaceutical aspects as well as aspects related to efficacy, safety, patient compliance and health care costs. But, not less important, are the analytical methodologies that can be used to guarantee the quality of biopharmaceutical medicines in the pre-clinical phase of development.

\section{References}

1. Hawe A, Wiggenhorn M, van de Weert M, Garbe JH, Mahler HC, et al. (2012)
Forced degradation of therapeutic proteins. J Pharm Sci 101: 895-913.

2. ICH (2006) Impurities in new drug substances

3. $\mathrm{ICH}(2006)$ Impurities in new drug products.

4. ICH (1995) Stability test of biotechnological/biological products. 